\title{
Ruxolitinib does not impair humoral immune response to COVID-19 vaccination with BNT162b2 mRNA COVID-19 vaccine in patients with myelofibrosis
}

\author{
Giovanni Caocci ${ }^{1,2}\left(10 \cdot\right.$ Olga Mulas $^{1,2} \cdot$ Daniela Mantovani $^{1} \cdot$ Alessandro Costa $^{2} \cdot$ Andrea Galizia $^{2} \cdot$ Luca Barabino $^{2}$. \\ Marianna Greco ${ }^{1} \cdot$ Roberta Murru ${ }^{1}$. Giorgio La Nasa ${ }^{1,2}$
}

Received: 21 June 2021 / Accepted: 10 July 2021 / Published online: 24 July 2021

(c) The Author(s), under exclusive licence to Springer-Verlag GmbH Germany, part of Springer Nature 2021

\section{Dear Editor,}

The COVID-19 pandemic has infected over 150,000 million people worldwide, causing approximately 3 million deaths, and a worldwide effort was made to develop efficient vaccines for this disease [1]. The BNT162b2 mRNA COVID19 vaccine has shown safety and efficacy, conferring $95 \%$ protection against COVID-19 in persons 16 years of age or older [2]. According to Italy's Strategic Plan for anti-SARSCoV-2/COVID-19 vaccination, BNT162b2 mRNA COVID19 vaccine has been proposed also for fragile patients [3]. Myelofibrosis (MF) is a clonal chronic myeloproliferative neoplasm (MPN) characterized by bone marrow fibrosis, progressive cytopenia, and extramedullary hematopoiesis. Anemia, infections, and progression to leukemia represent MF complications. MF patients are considered fragile, and thus eligible for COVID-19 vaccination, starting in March 2021, in Italy. According to the International Prognostic Scoring System (IPSS), patients with intermediate and high MF or other adverse risks may receive clinical benefits from ruxolitinib, the first approved JAK1/JAK2 inhibitor [4]. Ruxolitinib reduces the spleen volume and the circulating values of pro-inflammatory interleukins, in particular, IL-6 and TNF-alpha [5] and was shown to reduce the progression of acute respiratory distress syndrome (ARDS) and the need for respiratory assistance in COVID-19 patients [6][6]. Given the potent anti-inflammatory properties of ruxolitinib against immunocompetent cells [8], associated with an increased risk of viral and bacterial infection, we preliminarily explore

$\triangle$ Giovanni Caocci

giovanni.caocci@unica.it

1 Ematologia E CTMO, Ospedale Businco, ARNAS Brotzu, Cagliari, Italy

2 Dipartimento Di Scienze Mediche E Sanità Pubblica, Università Di Cagliari, Via Jenner, sn, 09124 Cagliari, Italy the efficacy of the humoral immune response to COVID-19 vaccination with BNT162b2 mRNA COVID-19 vaccine in MF patients either treated with ruxolitinib or other treatments. The study was conducted according to the guidelines of the Declaration of Helsinki.

The patient's characteristics are shown in Table 1. Overall, 20 patients (median age 66 years, range 48-82) suffering from MF were included in the study. A diagnosis of primary MF was performed in 13 cases (65\%), post-essential thrombocythemia MF in $6(30 \%)$ patients, and post-polycythemia vera MF in $1(5 \%)$ patient. Splenomegaly was present in 12 patients $(60 \%)$ and $13(65 \%)$ reported comorbidities. According to the DIPSS, 11 patients $(55 \%)$ were classified as low or intermediate-1 risk, and $9(45 \%)$ as intermediate- 2 or high risk. Molecular analysis was performed in all the patients: 15 out of 20 patients (75\%) were positive for the JAK2V617F and 5 (25\%) for CALR mutation. Over time, 10 patients $(50 \%)$ were receiving ruxolitinib, at a median total dose of $20 \mathrm{mg} / \mathrm{die}$ (range $20-40 \mathrm{mg}$ ), and the remaining 10 patients in other treatments ( 5 patients hydroxyurea and 5 only supportive therapy). None of the patients reported a previous COVID-19 infection.

All patients received 2 injections of $30 \mu \mathrm{g}$ per dose of BNT162b2 mRNA COVID-19 vaccine 3 weeks apart, according to the standard protocol. Participants gave written informed consent. After injection, mild pain at the injection site was frequently reported from the majority of the MF patients. No serious adverse events were registered. The serum level of IgG anti-Spike glycoprotein was tested after a median time of 45 days (range 40-60) from the second vaccine dose, using the approved anti-SARS-CoV-2 IgG CLIA (LIAISON® SARS-CoV-2 TrimericS IgG assay, DiaSorin, Saluggia, Italy). According to the manufacturer's recommendations, an arbitrary units per milliliter $(\mathrm{AU} / \mathrm{mL})$ ratio of $<12.0$ was considered to be negative, $12.0-15.0$ $\mathrm{AU} / \mathrm{mL}$ to be borderline, and $>15 \mathrm{AU} / \mathrm{mL}$ to be positive. $\mathrm{A}$ 
Table 1 Characteristics of 20 patients with myelofibrosis

\begin{tabular}{|c|c|c|c|}
\hline & Ruxolitinib & Other treatment & $p$ \\
\hline Age, median (range) & $66(48-72)$ & $70(52-82)$ & ns \\
\hline \multicolumn{4}{|l|}{ Disease, $\mathrm{N}^{\circ}(\%)$} \\
\hline Primary MF & $6(60)$ & $7(70)$ & \multirow[t]{3}{*}{ ns } \\
\hline Post-TE MF & $3(30)$ & $3(30)$ & \\
\hline Post-PV MF & $1(10)$ & 0 & \\
\hline \multicolumn{4}{|l|}{ Driver mutation, $\mathrm{N}^{\circ}(\%)$} \\
\hline JAK2 V617F & $7(70)$ & $8(80)$ & \multirow[t]{4}{*}{ ns } \\
\hline CALR & $3(30)$ & $2(20)$ & \\
\hline MPL & 0 & 0 & \\
\hline Triple negative & 0 & 0 & \\
\hline \multicolumn{4}{|l|}{ DIPSS, $\mathrm{N}^{\circ}(\%)$} \\
\hline Low/Int-1 & $3(15)$ & $8(40)$ & \multirow[t]{4}{*}{0.02} \\
\hline Int-2/High & $7(35)$ & $2(10)$ & \\
\hline Total dose of ruxolitinib mg/die, median (range) & $20(20-40)$ & & \\
\hline Time of exposition to ruxolitinib in months, median (range) & $27(3-112)$ & & \\
\hline Spleen $\mathrm{cm}$ below costal margin, median (range) & $6.7(0-21)$ & $2.4(0-14)$ & ns \\
\hline Hemoglobin g/dL, median (range) & $9.07(7.1-12.6)$ & $11.86(6.9-16.0)$ & 0.02 \\
\hline $\mathrm{WBC} \times 10^{3} / \mathrm{uL}$, median (range) & $10.77(1.7-68.7)$ & $13.1(2.8-72.0)$ & $\mathrm{ns}$ \\
\hline Platelets $\times 10^{3} / \mathrm{uL}$, median (range) & $242.4(17-840)$ & $379.4(28-1090)$ & ns \\
\hline LDH U/L, median (range) & $740(282-2539)$ & $319(144-563)$ & ns \\
\hline Humoral immune response achieved, $\mathrm{N}^{\circ}(\%)$ & $6(60)$ & $7(70)$ & ns \\
\hline IgG anti-Spike AU/mL, median (range) & $87.9(8.7-446.8)$ & $182.3(5-445)$ & ns \\
\hline \multicolumn{4}{|l|}{ Comorbidities, $\mathrm{N}^{\circ}(\%)$} \\
\hline Arterial hypertension & $3(30)$ & $4(40)$ & \\
\hline Occult hepatitis B infection & $3(30)$ & $1(10)$ & \\
\hline Chronic lymphocytic leukemia & 0 & $2(20)$ & \\
\hline Monoclonal gammopathy & $1(10)$ & 0 & \\
\hline Hypothyroidism & 0 & $2(20)$ & \\
\hline Diabetes & 0 & $1(10)$ & \\
\hline
\end{tabular}

conversion of $\mathrm{AU} / \mathrm{mL}$ to binding antibody units (BAU/mL) as recommended by the World Health Organization (WHO) guidelines is possible considering the following equation: $\mathrm{BAU} / \mathrm{mL}=2.6^{*} \mathrm{AU} / \mathrm{mL}$. Overall, a positive immune response against COVID-19 was achieved in 6 out of 10 patients $(60 \%)$ in the ruxolitinib group, in comparison with 7 out 10 patients $(70 \%)$ in the other treatment group ( $p=\mathrm{NS})$. Notably, the absolute IgG anti-Spike value was lower in the ruxolitinib group (median $87.9 \pm 136.6$ ) in comparison with the other group (median 182.3 $\pm 160.5 ; p=0.17$ ). Six out of $20 \mathrm{MF}$ patients (30\%) reported IgG anti-Spike humoral values considered not protective; in univariate analysis, any clinical or demographic variable was found associated with a lower humoral immune response to the vaccine.

In conclusion, we here report the first preliminary report on the safety and humoral efficacy of BNT162b2 mRNA COVID-19 vaccine in MF patients. Patients under ruxolitinib, a drug with a potential action against immunocompetent cells, achieved a protective humoral immune response similar to those patients who underwent other treatments.
The absolute IgG anti-Spike value was found lower in the group treated with ruxolitinib. Whether patients with a potential insufficient humoral response to vaccine or those with a lower response will benefit from a third dose of BNT162b2 mRNA COVID-19 vaccine is a matter of further investigation as well as the rate of future COVID-19 infections that will be registered in all patients considered in the present study. Our preliminary data need to be confirmed in larger cohort of MF patients.

\section{Declarations}

Ethics approval All procedures performed were in accordance with the ethical standards of the institutional committee and with the 1964 Helsinki declaration and its later amendments or comparable ethical standards.

Informed consent Written informed consent was obtained from all individual participants included in the study. 
Conflict of interest The authors declare no competing interests.

\section{References}

1. Carneiro DC, Sousa JD, Monteiro-Cunha JP (2021) The COVID19 vaccine development: a pandemic paradigm. Virus Res 301:198454

2. Polack FP, Thomas SJ, Kitchin N, Absalon J, Gurtman A, Lockhart S et al (2020) Safety and efficacy of the BNT162b2 mRNA Covid-19 vaccine. N Engl J Med 383(27):2603-2615

3. Salute M della. Guidelines of the strategic plan on COVID-19 vaccines approved by parliament [Internet]. [cited 2021 Jun 2]. Available from: https://www.salute.gov.it/portale/news/p3_2_ $1 \_1 \_1$.jsp? lingua $=$ italiano $\&$ menu $=$ notizie $\& \mathrm{p}=$ null $\& \mathrm{id}=5208$. Accessed 6 April 2021

4. Tefferi A (2016) Primary myelofibrosis: 2017 update on diagnosis, risk-stratification, and management. Am J Hematol 91(12):1262-1271
5. Arana Yi C, Tam CS, Verstovsek S (2015) Efficacy and safety of ruxolitinib in the treatment of patients with myelofibrosis. Future Oncol Lond Engl 11(5):719-733

6. Caocci G, La Nasa G (2020) Could ruxolitinib be effective in patients with COVID-19 infection at risk of acute respiratory distress syndrome (ARDS)? Ann Hematol 99(7):1675-1676

7. La Rosée F, Bremer HC, Gehrke I, Kehr A, Hochhaus A, Birndt $S$ et al (2020) The Janus kinase $1 / 2$ inhibitor ruxolitinib in COVID-19 with severe systemic hyperinflammation. Leukemia 34(7):1805-1815

8. Vannucchi AM, Sordi B, Morettini A, Nozzoli C, Poggesi L, Pieralli F et al (2021) Compassionate use of JAK1/2 inhibitor ruxolitinib for severe COVID-19: a prospective observational study. Leukemia 35(4):1121-1133

Publisher's note Springer Nature remains neutral with regard to jurisdictional claims in published maps and institutional affiliations. 